\title{
FRACTAL THERMODYNAMICS, BIG DATA AND ITS 3D VISUALIZATION
}

\author{
E.K. Paramonova ${ }^{1}$, A.N. Kudinov ${ }^{2}$, S.A. Mikheev $^{2}$, V.P. Tsvetkov ${ }^{2, a}$ \\ and I.V. Tsvetkov ${ }^{2}$ \\ ${ }^{1}$ GBUZ Tver region "Tver Regional Clinical Hospital", 170036, Tver, \\ Sankt-Petersburgskoe shosse, 105 \\ ${ }^{2}$ Tver State University, 170100, Russia, Tver, Zhelyabova, 33 \\ E-mail: ${ }^{a}$ Tsvetkov.VP@tversu.ru
}

\begin{abstract}
The article proposes to use fractal thermodynamics to analyze big data with fractal structure. The need for big data analysis arises in many areas of science and technology when creating databases in economics, medicine, geophysics, astronomy, particle physics and many others. This task is greatly simplified if big data has structural patterns. In this talk, we considered the case when big data forming a set $A$ with a high degree of accuracy are multifractals. The analysis of the fractal structure of the set $A$ was carried out on the basis of the fractal thermodynamics model. Its main methods and approaches are described. As a specific example of the set $A$, we consider the quantum phase spaces of the instantaneous heart rate $S_{q}$, built on the basis of big data on the $R R$-intervals of daily Holter monitoring of patients from the Tver Regional Clinical Hospital. It is shown that the sets $S_{q}$ are fractals with a high degree of accuracy and, therefore, the method of fractal thermodynamics is quite applicable for studying their structure.
\end{abstract}

Keywords: fractal thermodynamics, bigdata, quantum phase space, 3D visualization.

Ekaterina Paramonova, Alexei Kudinov, Sergey Mikheev, Viktor Tsvetkov, Ilya Tsvetkov 


\section{Introduction}

The need for big data analysis takes place in many areas of science and technology when creating databases in economics, medicine, geophysics, astronomy, particle physics and many others $[1,2]$.

This task is greatly simplified if big data has structural patterns. In this talk, we will consider the case when big data form a set $A$ with a high degree of accuracy are multifractals.

A multifractal can be represented as a union of homogeneous fractal subsets $A_{i}$ of the original set $A$, each of which has its own value of the fractal dimension $D_{i}, i=1,2, \ldots, I$.

The sets $A_{i}$ are fractal if, being embedded in the space $R^{n}$, then they are covered by elementary cubes of this space by the number $N_{i}$ with a power law dependence on the sizes of the sides $h$ of these cubes.

Consider the power functions $\bar{N}_{i}(h)=\Gamma_{i} h^{-D_{i}}$, which approximates the functions $N_{i}(h)$. Take a sequence of values $h_{k}, k=1,2, \ldots, K$. The degree of deviation $\delta$ of the functions $N_{i}(h)$ and $\bar{N}_{i}(h)$ is estimated by the formula:

$$
\delta_{i}=\max _{1 \leq k \leq K}\left|N_{i}\left(h_{k}\right)-\bar{N}_{i}\left(h_{k}\right)\right| / \bar{N}_{i}\left(h_{k}\right) .
$$

If $\delta_{i}$ is small, then the sets $A_{i}$ will be considered a fractals. In this case, the approximation parameters $D_{i}$ and $\Gamma_{i}$ represent the fractal dimensions of the sets $A_{i}$ and its $D_{i}$-dimensional fractal volums, respectively.

\section{Fractal thermodynamics}

The analysis of the fractal structure of the set $A$ will be carried out on the basis of the fractal thermodynamics model [3]. We briefly give its basic methods and approaches.

According to [3], we define the fractal entropy $S_{f}$ of the set $A$ by the formula $S_{f}=\ln \Gamma$.

Along with the fractal entropy $S_{f}$, we introduce into consideration the fractal temperature $T_{f}[3]$ :

$$
T_{f}=a \cdot\left(\frac{1}{n-D}-\frac{1}{n}\right)
$$

Coefficient $a$ in formula (2) for calculating the fractal temperature $T_{f}$ is selected for reasons of convenience in each specific case.

It follows from (2) that the fractal temperature $T_{f}$ is a function of the fractal dimension $D$ of the set $A$. From (2) it follows that for $D=0$ the value $T_{f}=0$.

Fractal thermodynamics, which we are developing, is one of the ways to study the properties of fractal systems and is an example of a more general concept of applying the methods of tropical mathematics to the problems of thermodynamics of various dynamical systems, proposed and developed by V.P. Maslov [4].

Since the fractal parameters $\Gamma$ and $D$ are related by relation (1), then between the fractal entropy $S_{f}$ and the fractal temperature $T_{f}$ there should be a functional dependence reflecting the structure of the fractal set $A$. The nature of this dependence determines the fractal equation of state (FES) of the set $A$. Due to the fact that $S_{f}=0$ at $T_{f}=0$, then we choose the specific form of the FUS in the form of a power function:

$$
S_{f}=b \cdot T_{f}^{\gamma} .
$$

The value of the index of the power function $\gamma$ in (5) will be called the FES index. 


\section{Fractal thermodynamics and quantum phase space of instantaneous heart rhythm}

As a specific example of the set $A$, consider the quantum phase spaces of instantaneous heart rhytm (IHR) $S_{q}$ [3], built on the basis of data on $R R$-intervals of daily Holter monitoring (HM) of patients from the Tver Regional Clinical Hospital (TRCH).

The HM database in TRCH was created in 2016, and its data is retained for ten years from the date its receipt. The total volume of data has already exceeded 80 terabytes. The Holter monitoring data of each patient by itself represent a rather large array of real-time data obtained from the Holter sensors. Our approach allows us to identify structural patterns in the series of raw HM data.

Our estimates of the parameter $\delta$ for $S_{q}$ for all patients considered in this work gave a value of no more than $1 \%$. Hence, it follows that with a high degree of accuracy the set $S_{q}$ is a fractal and, therefore, the method of fractal thermodynamics can be applied to the study of its structure.

In the database of the TRCH, a search was carried out for implementations of the 24-hours ECG for both patients with cardiac arrhythmias and students of Tver State University without significant cardiac arrhythmias. The study of these two categories of patients seems to be important for the development of methods for the early detection of cardiac rhythm pathologies.

The data obtained make it possible to construct a fractal diagram of the IHR states of the studied patients. It is shown in Fig. 1 as a graph with Cartesian axes $S_{f}$ and $T_{f}$. The fractal $S_{f} T_{f}$ state diagram allows one to visualize the nature of the functional dependence of $S_{f}$ on $T_{f}$. All patients are numbered with the index $p=1,2, \ldots, 19$, and the numbers $p=1_{1-3}$ and $p=2_{1-3}$ correspond to two patients with different dates of HM. The coefficient $a$ in the formula for calculating the fractal temperature (2) was taken equal to 10 .

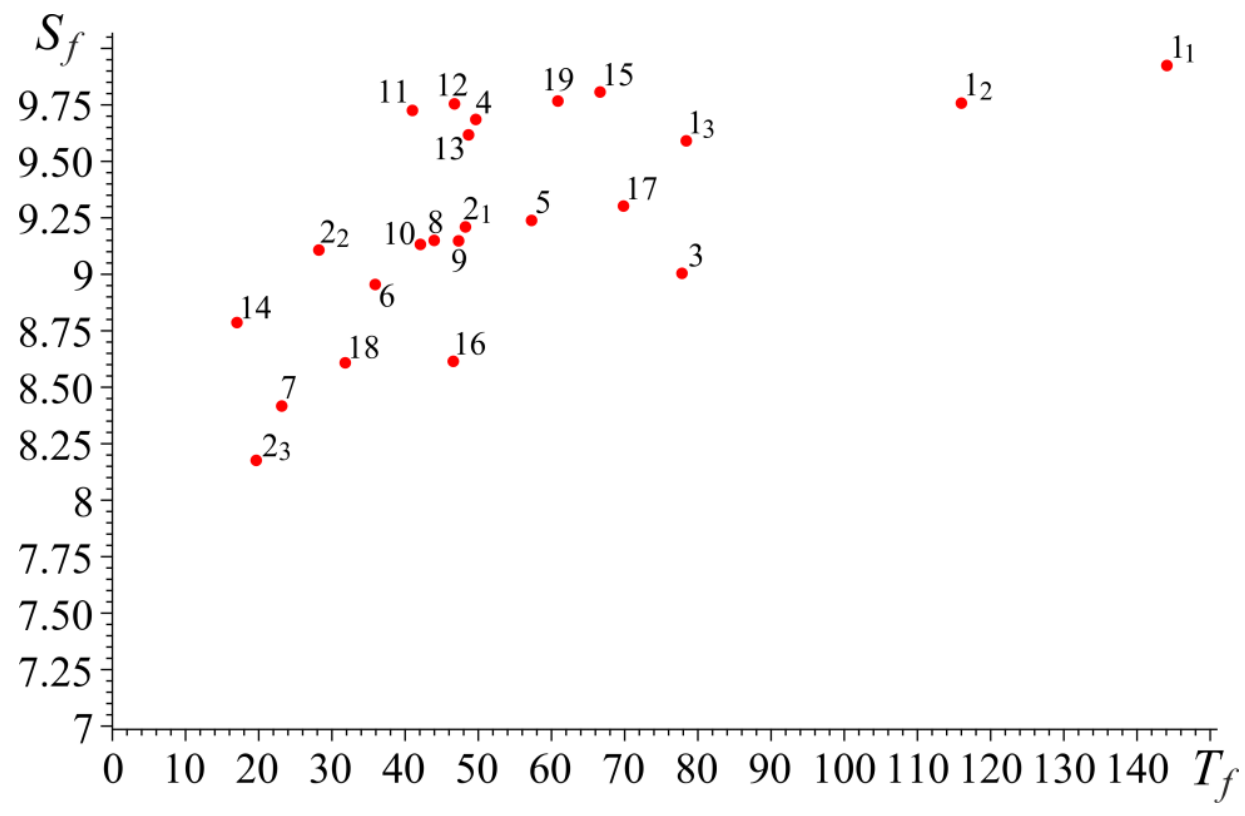

Figure 1. $S_{f} T_{f}$-diagram

The fractal diagram of state $S_{f} T_{f}$ allows visualizing the character of the functional dependence of $S_{f}$ on $T_{f}$. Figure 1 shows that all the states of the $S_{f} T_{f}$ diagram occupy a narrow band with a width of 1.3 and a length of 127 dimensionless units. 


\section{3D Visualization of the Quantum Phase Spaces of Instantaneous Cardiac Rhythm}

Let us construct a 3D histogram of the QPS of ICR as a pictorial presentation of its distribution by the states, which are described the discrete values of the IHR frequencies $y_{i}$, rate of change of frequencies $v_{i}$ and the occupation numbers $n_{i}$. The $y v$-plane projection of the 3D histogram of the QPS of ICR gives 2D visualization of the QPS of ICR, and its $y n$-plane and $v n$-plane projections represent the ICR frequency spectrum and the ICR frequency change rate spectrum. It is extremely sensitive information on the ICR state dynamics character.

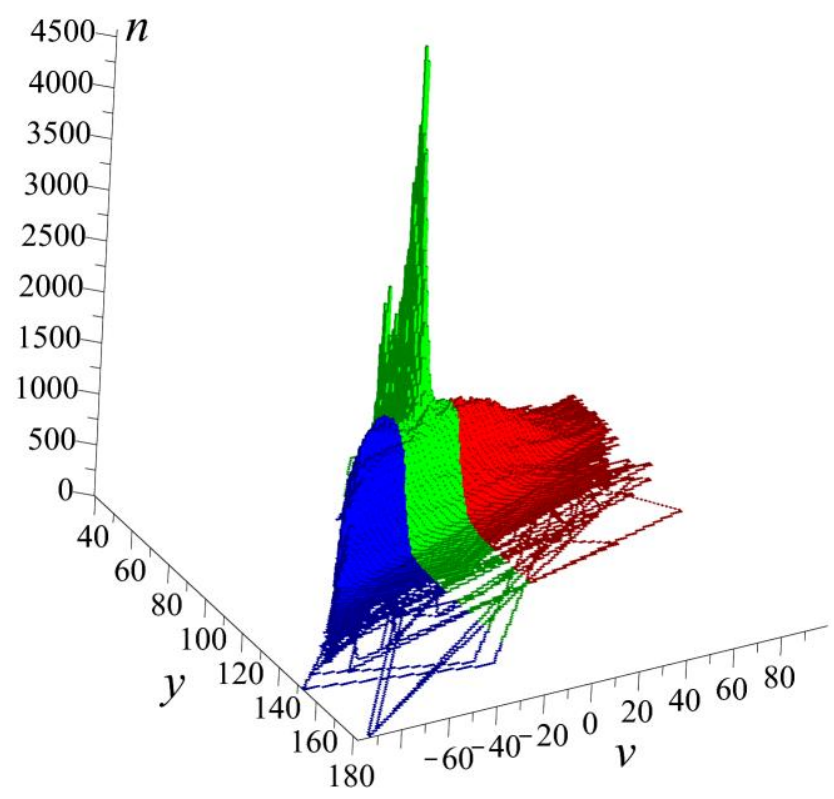

Figure 2. The 3D visualization of the QPS of ICR of the patient $p=10$; diagnosis: ventricular arrhythmia.

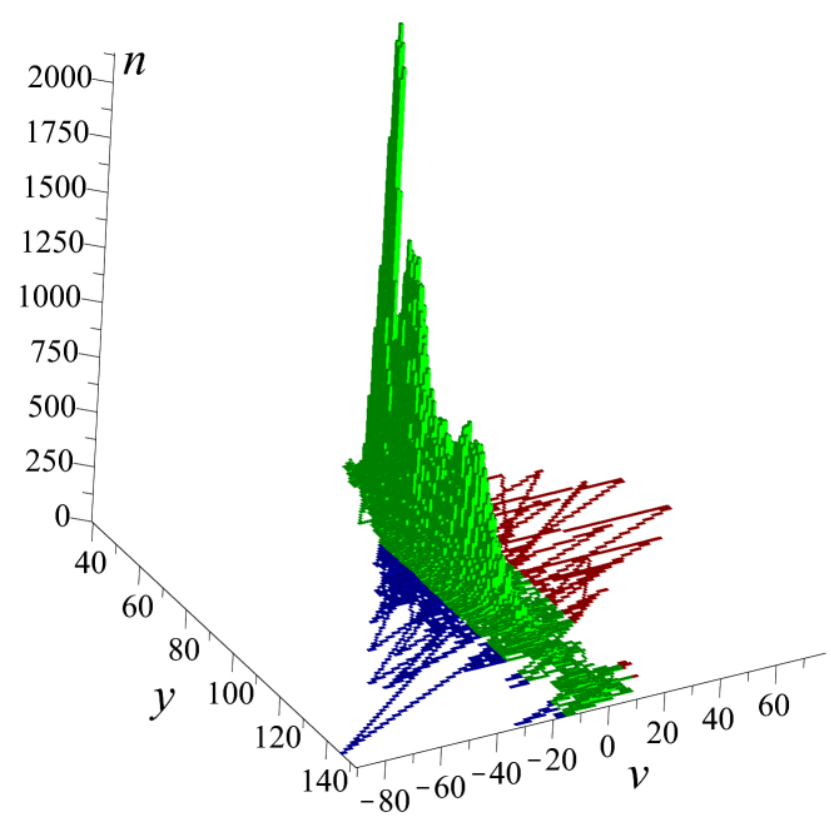

Figure 3. The 3D visualization of the QPS of ICR of the patient $p=7$; diagnosis: norm.

The 3D histogram of the QPS of ICR can be represented as a set of colored right-angled parallelepipeds in $R^{3}$ of side $n_{i}$ with square bases of side $h$ and the coordinates of their centers $y_{i}, v_{i}$. 
Fig. 2, 3 represent the 3D histograms of the QPS of ICR of patients $p=1,2$ of Tver Regional Clinical Hospital constructed using the Maple program system according to the data from 24-hour HM. The vertical planes $v= \pm 15$ divide $S_{q}$ into three areas $|v|\langle 15, v<-15, v\rangle 15$ [5]. The first area, corresponding to regular ICR, are displayed in green, the second area and third area, corresponding to ICR jumps (catastrophes) with an acceleration of the rhythm - in red, and with a deceleration of the rhythm - in blue.

\section{Conclusion}

The article shows the effectiveness of using fractal thermodynamics for analyzing big data with a fractal structure and is demonstrated by the example of the HM database in TRCH. The analysis of the fractal structure of the set A was carried out on the basis of the fractal thermodynamics model. Its main methods and approaches are described. As a specific example of the set A, we consider the quantum phase spaces of the instantaneous heart rate Sq, built on the basis of big data on the RRintervals of daily Holter monitoring of patients from the Tver Regional Clinical Hospital. It is shown that the sets Sq are fractals with a high degree of accuracy and, therefore, the method of fractal thermodynamics is quite applicable for studying their structure.

\section{Acknowledgement}

The authors thank VP Maslov for his support of works on fractal thermodynamics and valuable advice.

\section{References}

[1] Harding M., Hersh J. Big Data in economics // IZA World of Labor. 2018: 451. DOI: $10.15185 /$ izawol.451.

[2] Junichiro Hayano, Ken Kiyono, Emi Yuda, BS, Yoshiharu Yamamoto and Itsuo Kodama. Holter ecg big data project: allostatic state mapping by ambulatory ecg repository (ALLSTAR) // International Journal of Information Research and Review, 2018, V. 05, Issue, 07, pp. 5617-5624. URL: https://www.ijirr.com/sites/default/files/issues-pdf/3004.pdf

[3] Paramonova E.K., Mikheev S.A., Tsvetkov V.P., Tsvetkov I.V. Fractal Thermodynamics of the States of Instantaneous Heart Rhythm // Russian Journal of Mathematical Physics. 2021. V. 28. No. 2. pp. 251-256. DOI: 10.1134/S1061920821020096.

[4] Maslov V.P. Thermodynamics, Idempotent Analysis, and Tropical Geometry as a Return to Primitivism // Russian Journal of Mathematical Physics. 2016. V. 23. No. 2. pp. 278-280. URL: https://link.springer.com/content/pdf/10.1134/S1061920816020126.pdf

[5] Tsvetkov V.P., Mikheyev S.A., Tsvetkov I.V. Fractal phase space and fractal entropy of instantaneous cardiac rhythm // Chaos, Solitons and Fractals. 2018. V. 108. pp. 71-76. DOI: 10.1016/j.chaos.2018.01.030. 